\title{
COL-PMS: A Collaborative Performance Measurement System
}

\author{
María José Verdecho, Juan José Alfaro-Saiz, and Raúl Rodríguez-Rodríguez \\ Department of Business Organization, CIGIP (Research Centre on Production \\ Management and Engineering), Universidad Politécnica de Valencia, Camino de Vera, s/n, \\ 46022, Valencia, Spain \\ \{mverdecho, jalfaro, raurodro\}@cigip.upv.es
}

\begin{abstract}
Enterprises look for new strategies to increase/maintain their competitiveness. One of these strategies consists of establishing collaboration relationships with other enterprises. Collaborating enterprises need tools and methods that facilitate the management their performance. Performance Measurement Systems developed in the literature have overlooked the development of frameworks that include the social side of collaboration relationships under an integrated and structured approach. The purpose of this paper is to introduce the COLlaborative Performance Measurement System (COL-PMS) framework which overcomes this gap.
\end{abstract}

Keywords: performance measurement system, collaboration, interorganizational relationships.

\section{Introduction}

Global competition, higher specifications of products/services, and fast technological changes are some of the factors that are boosting enterprises to adopt new business models to remain competitive in the marketplace. In this context, some enterprises that traditionally operated with their suppliers under a transactional approach, have shifted their strategy and adopted collaborative initiatives. Collaboration involves two or more independent enterprises working together to align their processes with the goal of creating value to end customers and stakeholders with greater success than acting alone [1]. It implies sharing information, resources, responsibilities, knowledge, risk and profits among the partners [2]. In addition, in the current business environment, information and communication technologies play a fundamental role increasing the interoperability among the different enterprises and supporting the different processes and activities developed by the collaborating enterprises.

Performance measurement is 'a process of quantifying the efficiency and effectiveness of pass action' [3]. The complexity of collaboration environments requires new tools that aid to define and collect the necessary information for measuring the performance of enterprises. For that reason, Performance Measurement Systems (PMSs) should include in their structure special characteristics to support this issue. In 
supply chain management, interorganizational relationships rely on 'hard' (technological and infrastructural) and 'soft' (social and behavioural) aspects [4, 5]. Around $50 \%$ of interorganizational performance drivers are considered to rely on people factors while process and technology factors represent $30 \%$ and $20 \%$ respectively [6]. In fact, in real assessments of business relationships, 'hard' aspects have been addressed while 'soft' aspects appear to be overlooked [7]. For that reason, one of the lacks of PMS for collaborative contexts is the inclusion of mechanisms and elements that aid to manage jointly hard and soft aspects under an integrated framework.

The purpose of this paper is to introduce a PMS for collaborative contexts, called (COLlaborative Performance Measurement System (COL-PMS)), that fills this gap by including both (hard and soft) aspects within its structure in order to provide a tool for managing the performance of collaboration relationships more efficiently and effectively.

The structure of this paper is as follows. First, a literature review of PMS for interorganizational contexts is presented. Then, the COL-PMS framework is described. Finally, conclusions are exposed.

\section{Background}

The behavioral, social or relational side of collaboration consists of a set of aspects or elements that describe the interaction among the different partners. In [8], it is suggested that successful partnerships rely on three types of characteristics: attributes of the partnership (commitment, coordination, trust, etc.), communication behavior (such as information sharing) and conflict management techniques.

Based on [8], a literature review regarding PMS for interorganizational environments is performed to analyze if PMS developed support the behavioral side of collaboration. In [9], a PMS is proposed using the Balance Scorecard (BSC) [10] to measure supply chain (SC) performance. Their work presents the classical four perspectives (business process, customer, financial, and innovation and learning), generic goals of the SC and examples of performance indicators for these goals. Regarding the social elements, two of the goals to accomplish within the innovation and learning perspective are 'partnership management' and '(shared) information flows'. For these goals, the performance indicators 'product commitment ratio' and 'number of shared data sets with respect to total data sets' are defined. However, other aspects could be included to manage SC performance such as trust and commitment.

In [11], it is presented a framework for supply chain performance measurement that classifies metrics within three levels of management: strategic, tactical and operational. The authors also indicate the importance of measuring partnerships and present a list of partnership evaluation parameters such as 'level and degree of information sharing' and extent of 'mutual assistance in problem solving'. Nevertheless, the framework could be extended by considering other elements such as trust, coordination and conflict management.

In [12], it is developed a process-based PMS for supply chains. The work establishes a set of steps to decompose the core supply chain processes into lower level processes. The PMS associates goals, responsibility and function, and their respective performance measurements to each level and each element within each level 
(subprocesses, activities). It identifies that the strategy of the supply chain should be defined to identify the core processes. Nevertheless, social collaborative elements (information sharing, trust, etc.) can be intuited to be within the subprocesses and activity borders, but they are not explicitly defined.

In [13], it is proposed a PMS for Extended Enterprises (EEs). The PMS comprises three levels: enterprise, business unit and extended processes. The work considers strategic and operational coordinating measures which should include collaboration measures. The authors present a case study where the performance measurement of 'number of inter-partner strategic conflicts' is included in the EE scorecard. However, the work does not detail the extent of collaborative elements to be measured through the coordinating measures.

In [14], it is presented a PMS for EE. The PMS consists of two frameworks: a structural and a procedural framework. The structural framework comprises two levels: individual enterprise and EE PMS. At the individual level, each node PMS is composed of four perspectives: internal, supplier, customer and EE perspectives. However, the PMS could be further detailed by considering the social elements.

In [15], it is developed a PMS methodology for virtual enterprises. It defines two levels: virtual and individual enterprise. The work exposes the necessity to assess relationships within the PMS. The authors present a case study where the elements 'culture' and 'interpersonal relationships' are included in the 'virtual enterprise' and 'learning and growth' perspectives respectively but they do not specify how they are to be measured.

In [16], it is presented a PMS for supply networks composed of a methodology and a framework. The work identifies that equity and trust have to be maintained through the functional levels, perspectives and performance structure. However, the PMS does not consider how to integrate and measure these collaborative elements.

In [17], it is presented an audit model to assess an enterprise readiness for collaboration. Although the model is not structured as a PMS, it is considered within this group of the typology because it intends to evaluate the antecedent stage of a collaboration relationship. The model comprises four perspectives: strategic, operational, cultural and commercial. The cultural and commercial perspectives pursue to assess the compatibility of culture (trust, management style, information sharing, etc.) and commercial position (risk, investments, etc.) among enterprises. The work could be further extended by considering other social aspects (cooperation, coordination, conflict management, etc.).

In [18], it is developed a PMS for Virtual Organizations Breeding Environments (VBEs). The objective of a VBE is to prepare its member's organizations for their potential involvement in a collaborative opportunity-based virtual organization. The PMS is based on the four perspectives by Kaplan and Norton [19]. Collaboration elements are incorporated in all four PMS perspectives such as developing collaborative opportunities (outcome perspective), partnership development (stakeholder perspective), trust and relationship management (internal perspective), and culture, alignment of goals and collaboration (learning and growth perspective). However, although the work exposes the strategic map and performance indicators for measuring collaborative elements, a PMS structure is not explicit.

In [20], it is proposed a PMS for measuring collaboration performance in virtual organizations. The PMS is based on the four perspectives by Kaplan and Norton [10] 
and a fifth perspective for measuring collaboration that comprises five subperspectives: reliability, flexibility, responsiveness, commitment and communication. However, it would be necessary to explain further the extent of the five subperspectives and how these subperspectives are related to the rest of perspectives in order to define an integrated and solid PMS.

As a conclusion, it can be stated that although some of the PMSs reviewed include the measurement of some social elements in their structure, there is a clear lack of a collaborative PMS that considers the social side of the collaboration that allows managing the performance of collaborating enterprises under a solid performance structure. The framework proposed on this paper aims to fill this research gap.

\section{COL-PMS Framework}

\subsection{Description of COL-PMS}

From the literature review, it can be observed that there is a need of methods, systems and procedures that establish the steps to be followed to manage performance within collaborative contexts considering the social side of collaboration and following an integrated approach. The COL-PMS framework introduces these characteristics based on the PMS developed by Alfaro et al. [16] which is founded on three phases: 1) definition of the strategic framework, 2) definition of the process framework and 3) monitoring. The characteristics of a PMS for collaborative environments are related to the requirements that should be covered by the PMS in order to be considered solid and integrated. This implies that the PMS should provide all the necessary functionalities to approach the context for which it was developed. Additionally, this PMS should support the decision-making process of the enterprises and entities that collaborate. For that reason, it is necessary that the PMS considers two levels: interorganizational level (where collaboration takes place) and individual enterprise level. Both levels should be aligned in order to keep traceability among the performance elements that are to be defined.

At the individual enterprise level, COL-PMS derivates from the vision and strategy and reflects the most important aspects of the business. If this concept is extended within the interorganizational context, it can be said that it is a process of strategic planning for all the partners and implies a common understanding of their aims what facilitates the evaluation and degree of success reached in their objectives and strategies. Thus, COL-PMS starts with a strategic approach for its adequate interpretation and application. Therefore, the starting point of COL-PMS is the definition of the strategic framework (phase 1).

Figure 1 shows the composition of the COL-PMS generic framework which distinguishes between two types of frameworks: strategic and process framework. In detail, the definition of the strategic framework needs to incorporate all the performance elements (philosophical planning (mission and vision), stakeholder requirements, objectives, strategies, critical success factors and key performance indicators (KPIs). All these elements at defined for the four performance perspectives [10]: financial, customer, process and learning \& growth. These perspectives aid to structure performance measurement following relationships of cause-effect. 


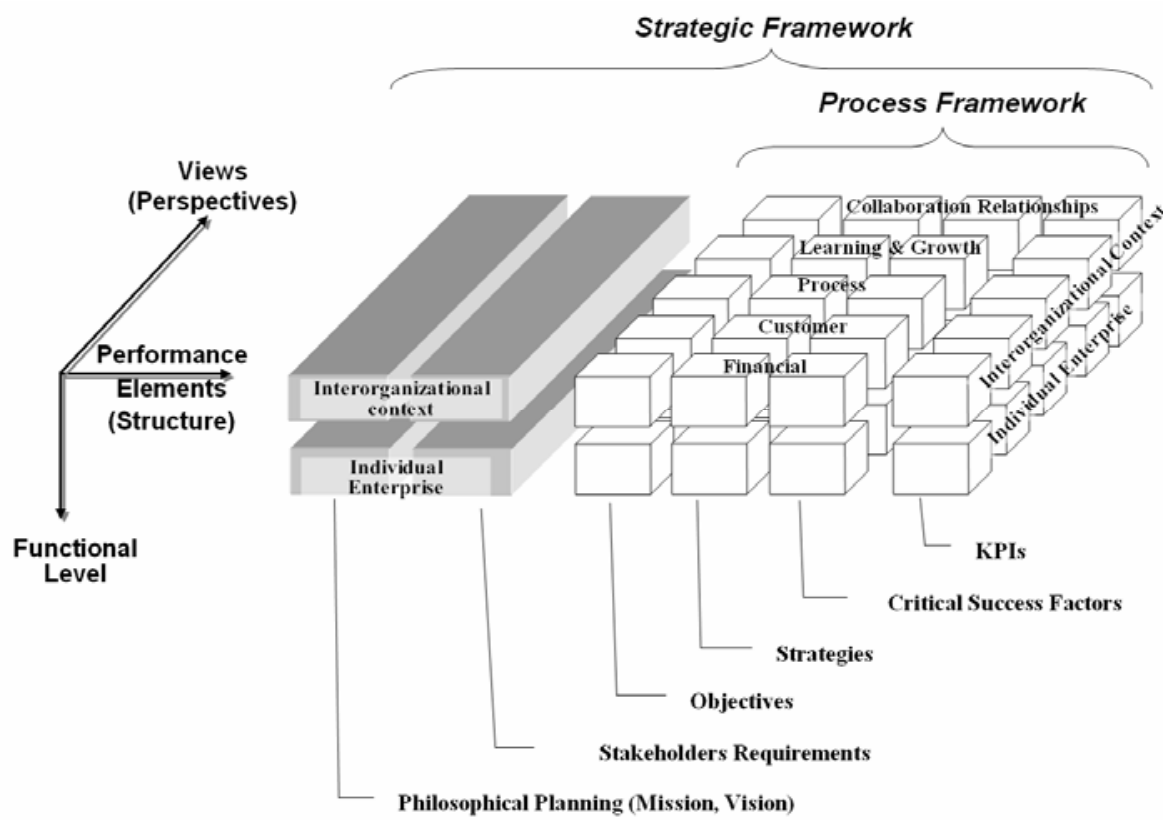

Fig. 1. COL-PMS Framework

In addition, it is necessary to introduce one perspective oriented to manage collaboration relationships that covers the social side of collaboration. This perspective supports the consecution of the other four perspectives, due to the fact that is related to the aspects of the collaborative culture which provides a mediator effect or social climate above the components of the rest of perspectives. Therefore, this perspective will consist of a set of objectives, strategies, critical success factors and KPIs related to relational characteristics such as the ones described by [8]: relationship attributes (trust, commitment, cooperation, etc.), communication behaviour (information sharing) and conflict resolution techniques. The descriptions of these characteristics are as follows (Boddy et al., 2000; Hanfield and Betchel, 2004; Lejeune and Yakova, 2005):

Commitment refers to the willingness of the supplier to perform effort on behalf of the relationship. It is the establishment of the foundation of the relationship and it is based on being supportive in solving problems together. A high level of commitment provides the context for the achievement of individual and mutual goals. There are different types of commitment depending on the effort (to continue the relationship), loyalty, contract duration or their combination.

Coordination involves the tasks that are to be taken for linking activities performed by the different members in a seamless manner. The coordination degree between manufacturer and supplier is an important attribute of the relationship as it allows moving together towards the achievement of mutual objectives.

Trust is based on the belief that the partner is reliable and will fulfill its responsibilities acting fairly. A partner trusts another partner if considers that decisions made by this last one will be in the interest of both parts. There are different types of trust 
depending if they are founded on deterrence, reliability, competence, goodwill, loyalty or their combination.

Information sharing considers the timeliness, accuracy, adequacy and completeness of the relevant information exchanged. It is the degree of private information that enterprises share in order to perform their activities and make decisions. It is one of the main flows through the companies and it defines the depth and width of the other two main flows (material and monetary) and develops the flow of social relationships.

Finally, conflict management measures the degree of intensity and conflict resolution mechanisms that exist between manufacturer and supplier. The existence of conflict is inherent to interpersonal as well as interorganizational relationships. However, the manner this conflict is managed is essential to the long-term and stability of the relationship.

It is not the purpose of this paper to provide a full overview of social characteristics of collaboration relationships but to present the elements that a PMS should have to manage them. For that reason, the social characteristics described are only given as examples that are to be considered but do not conform a definitive list.

In addition, it has to be noted that collaboration relationships perspective is mainly related to the interorganizational functional level as it is the level in which interorganizational collaboration takes place. However, social aspects between companies are not isolated from the individual enterprise level as it happens with the other four perspectives. Therefore, the consecution of the interorganizational level is linked to the individual enterprise level.

On the other hand, in the definition of the process framework (phase 2), it is not required to incorporate the philosophical elements (mission and vision) or the stakeholder requirements, excepting special circumstances that require it. Once the strategic framework is obtained, performance elements of the process framework (objectives, strategies, critical success factors and KPIs) are defined for those key business processes associated to the collaborative context. These key processes are processes directly linked to a common product/service produced by the partners or processes that support the success of the production of those products/services.

In the previous phases, all the performance elements have been defined. This last phase aims at monitoring of all those elements in order to know which are the most important elements, what levels are the most relevant from a performance management point of view and where are located special indicators for a specific partner. For that reason, it is convenient show graphically the deployment of all the elements by elaborating two types of diagrams: graphics of global deployment and graphics of partial deployment.

The graphics of global deployment represent the deployment of the basic performance measurement elements for all the functional levels (interorganizational context level and individual enterprise level). Consequently, there will be one graph for the objectives, another for the strategies, another for the critical success factors and, finally, another for the key performance indicators. Then, such graphics will also be constructed for both the strategic and the process framework. Figure 2 shows an example of graphic of global deployment for the objectives. 
Objectives

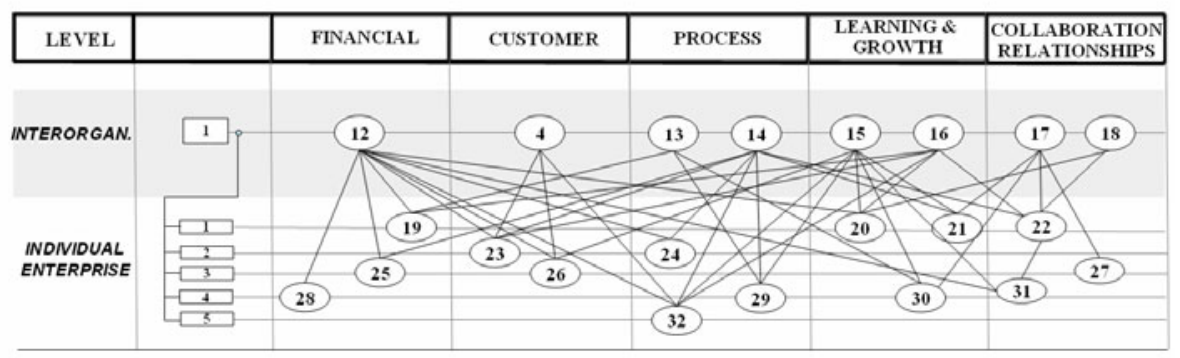

Fig. 2. Graphic of Global Deployment: Objectives

The graphics of partial deployment complements the graphics of global deployment providing the combination of elements necessary for measuring performance for each level (interorganizational level and individual enterprise level).

\section{Conclusions}

This paper has reviewed the literature regarding interorganizational PMSs in order to analyse if the social aspects of collaboration are managed through a solid and integrated PMS structure. Based on the gaps coming from the literature review, we have introduced a new collaborative PMS, called COL-PMS, which includes the social side of collaboration within its structure in order to provide a tool for managing the performance of collaboration relationships more efficiently and effectively.

This framework considers five performance perspectives in order to manage collaborative performance. In fact, there is a need to consider a specific perspective for managing the relational aspects of collaboration relationships as they act as a mediator element for reaching the elements that compose the other performance perspectives. In addition, we have described the elements that integrate the COL-PMS framework so that enterprises that desire to collaborate or are collaborating have a tool for aiding to define and collect performance management information.

Acknowledgments. This work has been developed within the framework of a research project funded by the Polytechnic University of Valencia, titled "Design and Implementation of Performance Measurement Systems within Collaborative Contexts for aiding the Decision-making Process", reference PAID-06-08-3206.

\section{References}

1. Simatupang, T.M., Wright, A.C., Sridharan, R.: Applying the theory of constraints to supply chain collaboration. Supply Chain Management: An International Journal 9(1), 57-70 (2004)

2. Mentzer, J., Min, S., Zacharia, Z.G.: The Nature of Interfirm Partnering in Supply Chain Management. Journal of Retailing 76(4), 549-568 (2001)

3. Neely, A., Adams, C., Kennerley, M.: The Performance Prism: The Scorecard for measuring and managing business success. Financial Times Prentice Hall, London (2002) 
4. Harland, C.M.: Supply Chain Management: Relationships, Chains and Networks. British Journal of Management 7, 63-80 (1996)

5. Burgess, K., Singh, P.: A proposed integrated framework for analysing supply chains. Supply Chain Management: An International Journal 11(4), 337-344 (2006)

6. Zaklad, A., McKnight, R., Kosansky, A., Piermarini, J.: The social side of the supply chain. Industrial Engineer 36(2), 40-44 (2004)

7. Staughton, R., Johnston, R.: Operational performance gaps in business relationships. International Journal of Operations \& Production Management 25(4), 320-332 (2005)

8. Mohr, J., Spekman, R.: Characteristics of partnership success: Partnership attributes, communication. Strategic Management Journal 15(2), 135-152 (1994)

9. Brewer, P.C., Speh, T.W.: Using the balanced scorecard to measure supply chain performance. Journal of Business Logistics 21(1), 75-93 (2000)

10. Kaplan, R.S., Norton, D.P.: The balanced scorecard - measures that drive performance. Harvard Business Review 70(1), 71-79 (1992)

11. Gunasekaran, A., Patel, C., Tirtiroglu, E.: Performance measures and metrics in a supply chain environment. International Journal of Operations \& Production Management 21(1-2), 71-87 (2001)

12. Chan, F.T.S., Qi, H.J.: Feasibility of performance measurement system for supply chain: a process-based approach and measures. Integrated Manufacturing System 14(3), 179-190 (2003)

13. Bititci, U.S., Mendibil, K., Martinez, V., Albores, P.: Measuring and managing performance in extended enterprises. International Journal of Operations \& Production Management 25(4), 333-353 (2005)

14. Folan, P., Browne, J.: Development of an Extended Enterprise Performance Measurement System. Production Planning and Control 16(6), 531-544 (2005)

15. Chalmeta, R., Grangel, R.: Performance measurement systems for virtual enterprise integration. International Journal of Computer Integrated Manufacturing 18(1), $73-84$ (2005)

16. Alfaro, J.J., Ortiz, A., Rodríguez, R.: Performance measurement system for Enterprise Networks. International Journal of Productivity and Performance Management 56(4), 305-334 (2007)

17. Bititci, U.S., Turner, T., Mackay, D., Kearney, D., Parung, J., Walters, D.: Managing synergy in collaborative enterprises. Production Planning \& Control 18(6), 454-465 (2007)

18. Romero, D., Galeano, N., Molina, A.: A conceptual Model for Virtual Breeding Environments Value System. In: Camarihna-Matos, L., Afsarmanesh, H., Novais, P., Analide, C. (eds.) Establishing the Foundation of Collaborative Networks. Springer, Boston (2007)

19. Kaplan, R.S., Norton, D.P.: Strategy maps: converting intangible assets into tangible outcomes. Harvard Business School Publishing Corporation, Boston (2004)

20. Westphal, I., Thoben, K.D., Seifert, M.: Measuring collaboration performance in virtual organizations. In: Camarihna-Matos, L., Afsarmanesh, H., Novais, P., Analide, C. (eds.) Establishing the Foundation of Collaborative Networks. Springer, Boston (2007)

21. Boddy, D., Macbeth, D., Wagner, B.: Implementing collaboration between organizations: an empirical study of supply chain partnering. Journal of Management Studies 37(7), 1003-1018 (2000)

22. Handfield, R.B., Bechtel, C.: Trust, power, dependence, and economics: can SCM research borrow paradigms? International Journal of Integrated Supply Chain Management 1(1), 3 $32(2004)$

23. Lejeune, M.A., Yakova, N.: On characterizing the $4 \mathrm{C}$ 's in supply chain management. Journal of Operations Management 23(1), 81-100 (2005) 